\title{
Isobaric Tags for Relative and Absolute Quantitation 4-plex
}

National Cancer Institute

\section{Source}

National Cancer Institute. Isobaric Tags for Relative and Absolute Quantitation 4-plex. NCI Thesaurus. Code C161868.

An isobaric tag for relative and absolute quantitation reagent preparation containing four distinct isotope substitutions. 